\title{
INTERPRETANDO EL PROCESO \\ DE MULTICULTURALIDAD EN ESPAÑA Y LA PROPUESTA DE CIUDADANÍA CÍVICA DE LA UE*
}

\author{
Ricard Zapata-Barrero \\ Universidad Pompeu Fabra
}

\begin{abstract}
We are living an Historical Time characterized by the international migrations. The arrival and permanence of people coming mainly from developing countries increasingly make clear that the current instruments to manage conflicts coming from modernity have difficulties to work with normality. Following the approach of change of paradigm of modernity as interpretation approach (1), I emphasize the three main indicators of multiculturality in Spain: use of populist arguments on the part of traditional political parties, bond between two processes of multiculturalidad: the multinationality and immigration, secularism or the management of religious pluralism (2). My final intention (3) is to underline how this debate also helps us to identify a new fracture within the UE between one more an opened (innovation) and closed (tradition) policy of immigration. In the end I will do a final balance transferring this dilemma between tradition and innovation in the new frame of immigration policies: the Hague program initiated in November of 2004.
\end{abstract}

KEY WORDS Multiculturalism, citizenship, European Union, immigration, change process, innovation, tradition.
RESUMEN Estamos viviendo un Tiempo Histórico caracterizado por las migraciones internacionales. La llegada y permanencia de personas procedentes mayoritariamente de países en vías de desarrollo ponen cada vez más en evidencia que los instrumentos habituales para gestionar conflictos procedentes de la modernidad tienen dificultades para funcionar con normalidad. Siguiendo el enfoque de cambio de paradigma de la modernidad como enfoque para la interpretación (1), destaco los tres principales indicadores de multiculturalidad en España: uso de argumentos populistas por parte de partidos políticos tradicionales, vínculo entre dos procesos de multiculturalidad: la multinacionalidad y la inmigración, secularismo o la gestión del pluralismo religioso (2). Mi propósito final (3) es destacar cómo este debate nos ayuda también a identificar una nueva fractura dentro de la UE entre una política más abierta (innovación) y más cerrada (tradición) de inmigración. Al final haré un balance final trasladando este dilema entre tradición e innovación en el seno del nuevo marco de políticas de inmigración: el programa de la Haya, iniciado en noviembre de 2004.

PALABRAS CLAVE Multiculturalidad, ciudadania, Unión Europa, inmigración, proceso de cambio, innovación, tradición.

\section{Proceso de cambio de paradigma de la modernidad: rediscutiendo el principio del nacimiento}

Estamos viviendo un Tiempo Histórico caracterizado por las migraciones internacionales. La llegada y permanencia de personas procedentes mayoritariamente de países en vías de desarrollo ponen cada vez más en evidencia que los instrumentos habituales para gestionar conflictos procedentes de la modernidad tienen dificultades para funcionar con normalidad. En este caso, la forma moderna de gestionar la inmigración tiene muchas similitudes a un proceso de cambio de paradigma basado en el vínculo sagrado o la Santísima Trinidad del Estado/Nación/Ciudadanía. Como en la época de B. Constant, debemos, pues, poner al menos el mismo signo de interrogación a nacionalidad igual que antes lo pusimos a la pertenencia a la aristocracia. Hoy en dia el "mito de la sangre azul» se llama "ser francés de souche», "ser español de origen", "ser $X$ de nacimienton. Este es un signo de la Edad Media actual, cuando percibimos nuestra sociedad democrática históricamente. Todos estos argumentos muestran que discutir sobre fenómenos ligados a la multiculturalidad y a la ciudadanía tiene un carácter urevolucionarion indudable, de proceso de cambio de paradigma. Si con el paso de la época medieval a la época moderna, uno de los mayores logros de nuestro tiempo histórico fue que el nacimiento dejara de ser el fundamento y criterio explicativo básico de las desigualdades (entendiendo por nacimiento 
no sólo el hecho físico de nacer, sino el hecho de poseer caracteristicas físicas, culturales o simplemente del carácter que no dependen de mi voluntad), la situación que viven los inmigrantes significa un retorno a la edad media. Es como volver a ojear nuestros libros de historia, pero a través de las noticias de los telediarios y las portadas de los diarios. El principio del nacimiento vuelve a tener un significado social sin precedentes, traducible en tener o no derechos, en poder tener o no una identidad pública, en poder tener o no oportunidades de ascenso social, en poder beneficiarse o no de todos los e la democracia y del liberalismo (R. ZapataBarrero, 2004). En este marco, el vínculo entre ciudadanía y nacionalidad constituye la principal unidad de análisis generadora de la mayoría de los argumentos que animan los debates actuales (ciudadanía diferenciada, ciudadania transnacional, ciudadanía posnacional, ciudadanía múltiple, etc.). ${ }^{1}$

Este contexto nos permite establecer las premisas que me parecen fundamentales para orientar una interpretación adecuada del proceso. ${ }^{2}$

Como primera premisa debemos asumir que los debates sobre si estamos a favor o en contra del proceso son discusiones de última década del siglo $\mathrm{XX}$, similares a los que se producian en los inicios del proceso de globalización. Hoy en día mantener esta forma de argumentación es seguir un debate obsoleto. Igual que el proceso de globalización, este proceso de construcción de sociedades multiculturales es irreversible. Ir en contra es signo de estar fuera de nuestro tiempo histórico. Por lo tanto, las reflexiones que se hagan deben evitar partir de falsas premisas. La multiculturalidad no es un ideal a alcanzar, sino una realidad a gestionar. La cuestión es debatir cómo gestionar el proceso.

En este marco existen dos posibles reacciones. Por un lado, un lenguaje conservador que defiende un tipo re-activo de políticas, sólo concentrado en la construcción de muros de contención, de negación de que estamos en un proceso profundo de cambio. Por otro lado, un lenguaje progresista que apoya politicas pro-activas, que busca acomodar esta realidad dentro de la sociedad con su consecuente modificación. Estas dos lógicas tienen un carácter práctico evidente. Por ejemplo, ante el dato de que la ciudadanía vincula en un $65 \%$ la inmigración y la delincuencia, podemos seguir dos lógicas de interpretación. Una re-activa y conservadora. La que interpreta el dato como una realidad y que buscará impedir la llegada de más inmigrantes para evitar incrementar el porcentaje. Esta primera lógica interpreta el proceso en términos más cuantitativos que cualitativos. Una segunda lógica es plantearnos rápidamente la necesidad de hacer política educativa, asumiendo que este dato no representa la realidad, sino una interpretación de la realidad rodeada de estereotipos y de pre-juicios. En este caso se sigue una lógica progresista y pro-activa.

Como segunda premisa, este proceso tiene un efecto espejo que requiere una adecuada gestión ya que se mezclan razones y emociones. Lo cierto es que con la introducción de este tema, la sociedad española en general, la ciudadanía en particular, está en permanente estado de revisión de sus conciencias y de sus valores. Gestionar este efecto espejo es una de las claves para construir discursos y legitimar políticas.

Como tercera premisa, un marco de interpretación requiere percibir el proceso como oportunidad, y no como conflicto. La concepción del proceso de multiculturalidad como conflicto contra nuestros valores democráticos y liberales es una lógica de interpretación que en lugar de mantener la unidad, crea fragmentación y división. Convertir este proceso en oportunidad para profundizar en los valores y principios que actualmente articulan nuestras sociedades es uno de los principales objetivos que debe orientar toda interpretación del proceso. Lo contrario puede ser muy perjudicial para nuestra democracia.

Como cuarta premisa, existen dos formas de comenzar una interpretación del proceso: una que lo mide todo en términos instrumentales de beneficios económicos para nuestra sociedad, y otra que establece como prioridad el respecto de los derechos humanos. La primera lógica, aunque sea con buenas intenciones, debe limitarse con los principios de los derechos humanos, el lenguaje más adecuado a la realidad para gestionar el proceso. Por lo tanto la lógica instrumental de la interpretación de la inmigración debe siempre pasar el filtro de los derechos humanos. Lo contrario puede ser de nuevo contraproducente puesto que reducimos el numero de argumentos democráticos que justifiquen nuestra forma de gestionar el proceso.

Teniendo este marco de interpretación, veamos cuáles son los principales indicadores del proceso en España.

\section{Indicadores de multiculturalidad en España}

Existen muchos temas que pueden servir de indicador para analizar el proceso de cambio en España debido a la llegada

ARBOR CLXXXI 713 MAYO-JUNIO [2005] 101-114 ISSN: 0210-1963 
y permanencia de la inmigración. Destacaría al menos tres grandes temas: uso de argumentos populistas por parte de partidos políticos tradicionales; vinculo entre dos procesos de multiculturalidad: la multinacionalidad y la inmigración; y secularismo o la gestión del pluralismo religioso. Me ocuparé luego del marco de la UE y me plantearé cómo interpretar la propuesta de ciudadanía cívica de la Comisión teniendo cuenta estos indicadores de España como marco de reflexión.

\subsection{Uso de argumentos populistas por parte de los partidos tradicionales}

La "maquinaria del populismo" se nutre del vinculo entre el lenguaje de la seguridad, el socioeconómico y el de la identidad. Si nuestras democracias no tienen recursos suficientes para gestionar temas de multiculturalidad dejan un campo muy abierto a los nuevos populismos. Es una realidad que estos partidos tienen el proceso histórico de multiculturalidad como principal fuente para construir sus discursos. Se dirigen principalmente en contra del proceso mismo. Tanto los discursos como las movilizaciones de estos partidos, su penetración en los sistemas políticos tradicionales (a través de partidos, pero también de argumentos dentro de los partidos tradicionales), e incluso, sus auténticas fuerzas como alternativa de poder, es una "realidad realn, si se me permite la redundancia. Debemos considerar este auge como formando parte del proceso mismo de multiculturalidad, y por lo tanto como formando parte de los indicadores a analizar. ${ }^{3}$ La cuestión no sólo afecta al sistema político, sino también a nuestras tradiciones políticas. El populismo tiene también su campo de cultivo cuando las tradiciones liberales y democráticas no tienen recursos para gestionar el proceso. El populismo ofrece soluciones, soluciones simples, pero para la ciudadanía, son soluciones al fin y al cabo. Elabora un lenguaje que subraya aspectos implícitos de nuestra tradición basada en el vínculo Estado-nación-ciudadanía. Los discursos populistas se presentan como los nuevos cruzados (P. Perrinau, 2001), enarbolando la bandera de la Santisima Trinidad.

En España podemos decir que el discurso de la seguridad y el socioeconómico son los que priman. Todavía no se ha construido un discurso basado en la identidad. Por el momento, para el gobierno central, el peligro máximo que debe evitarse con el proceso de multiculturalidad en general, la inmigración en particular, es la inseguridad ciudadana que se genera en términos físicos, de espacios vitales, y socioeconómicos. La existencia de estos dos discursos no es distintivo en comparación con otros paises europeos.
Lo que sí constituye un aspecto diferenciador es que todavía no se hayan vinculado al tema de la identidad (española, catalana, vasca, etc.), sobretodo porque si bien los diferentes gobiernos podrian ponerse de acuerdo con la interpretación del proceso en términos de seguridad y socioeconómicos, seguro que contribuirá más a la fractura social su interpretación del proceso en términos de efectos sobre sus identidades comunitarias. En otros paises, como en Francia (Holanda, Austria, Bélgica, Italia) el discurso de la identidad y el discurso de la seguridad, en su versión extremista, está monopolizada por el partido del Frente Nacional (Front National, el nombre ya nos da bastante información sobre sus intenciones). El populismo español, por el momento, y si nos atenemos a sus versiones muy embrionarias de localidades catalanas, no tiene directamente el discurso identitario como señal, sino la seguridad en el bienestar de las personas. Utiliza como principal núcleo para producir sus argumentos las zonas de contacto entre la población inmigrante y la población autóctona, sobretodo alimentada por la idea de que también son zonas de conflicto. Es un populismo, podriamos decir, que avanza hacia el electorado de izquierdas, puesto que sus principales votantes son los que tradicionalmente apoyan a estos partidos tradicionales, especialmente socialistas, aunque también de tradición comunista. El populismo en España puede hacer más daño electoral a la izquierda tradicional que a los partidos de derechas tradicionales. Su discurso es en torno a los beneficios del sistema de bienestar, y de la visión de competencias que los ciudadanos de los barrios tienen. No es todavía un populismo de derechas, esto es, centrado en la identidad patriótica nacional. Este tipo de populismo todavia no ha brotado en España de forma explícita, seguramente por la misma razón que tampoco lo ha hecho en Alemania: por la memoria histórica que supondría volver a presenciar una época con partidos que pensábamos habian desaparecido definitivamente de la escena del sistema político y como alternativa de poder. Aunque también en este punto existe un rasgo distintivo español que otros partidos de derechas europeos están adoptando: el hecho de incorporar dentro del discurso sobre la inmigración la mayoría de los argumentos relacionados con la seguridad que correspondería a los partidos populistas. Desde un punto de vista de político la estrategia es eficaz: para evitar que broten partidos populistas por la derecha, o bien que se conviertan en auténticas alternativas de poder, como en Francia, Holanda, y Austria, lo mejor es adoptar sus discursos, pero suavizando su vocabulario y mensaje radical. En cualquiera de los casos, estos partidos populistas frenan el proceso de multiculturalidad 
y consideran que el proceso mismo tiene sólo dimensiones negativas con consecuencias directas sobre nuestra calidad de vida y nuestra identidad.

Pero lo que también importa es que tras las diferentes convocatorias electorales (locales, autonómicas, estatales) durante el 2003 y el 2004, los partidos políticos en España están construyendo sus discursos sobre la inmigración. Este momento es clave. El diseño de este discurso tiene unas implicaciones sociales muy directas, puesto que lo que se está discutiendo son argumentos que adquieren el carácter de principios prácticos para el ciudadano, quien necesita justificar sus percepciones y comportamientos ante este proceso de cambio en el que nos encontramos y que tiene su origen en la llegada y permanencia de la inmigración (G. Aubarell y R. Zapata, eds. 2003). Al construir discursos, los partidos están legitimando comportamientos ciudadanos, y confirmando/negando interpretaciones del proceso que tienen más un origen emocional que racional.

En este contexto es muy importante que el discurso político genere un marco de interpretación del proceso que evite el populismo. Estoy hablando no sólo de contenidos, sino de formas. El populismo como estilo discursivo de hacer política. Sabemos que una de las características del tema de la inmigración es que se puede apelar muy fácilmente al "pueblo», a la ciudadanía, para justificar decisiones. En estos casos prevalece más una forma populista de argumentación. El gran desafío que tienen los partidos tradicionales de nuestro pais no es que se creen partidos con discursos antiinmigrantes, sino que la forma de argumentación que tengan sean populistas. El populismo está penetrando por la "puerta trasera", es decir, dentro de partidos tradicionales del sistema político y sin uruido» ni con «mala intención», de forma casi inconsciente, pero debemos detenernos todos y pensar en los efectos.

En el momento en que la percepción que tiene la ciudadanía prevalece como único criterio legitimador de discursos políticos, estamos en pleno círculo semántico del populismo. Si la argumentación populista se enrolla narcisicamente en el comportamiento discursivo de los partidos políticos, se puede contribuir más al conflicto que a la cohesión, al descontrol que a la estabilidad. Si el político da al ciudadano lo que el ciudadano quiere escuchar, no lo que debe escuchar, se está fomentando precisamente lo que se debe evitar. Considerar lo que piensa el ciudadano como excusa para justificar injusticias es un extremo que debemos evitar.
El peligro democrático hoy en dia no es que aparezcan partidos populistas como respuesta a la desorientación que provoca procesos de cambio originados por la inmigración, sino que los partidos tradicionales se "popularicen", si me permiten la expresión, a través de sus comportamientos discursivos y que pierdan un estilo de hacer política donde sigan prevaleciendo como criterios la igualdad y la justicia. Lo que piensa la ciudadanía, su interpretación de la inmigración, no son criterios democráticos de justicia, ni deben ser los que orienten discursos. Lo que debe ser objeto de reflexión para diseñar el discurso político de la inmigración es plantearnos cómo es posible que la ciudadania tenga dificultades de asumir el cambio que se está produciendo en sus barrios con la llega de inmigrantes, qué políticas hacer para que no se sientan desatendidos y sean permeables al discurso populista. Apelar a la ciudadanía cuando no se tienen argumentos es puro cinismo político en el sentido más clásico, que puede tener efectos contrarios (legitimar percepciones estereotipadas y fomentar la división social entre inmigrantes y ciudadanos), incluso cultivar emociones hasta llegar a la "caza al inmigrante», "el inmigrante como causa de todos los males".

Los políticos, ante la desorientación que provoca el proceso en el que estamos, no pueden tomar la vía fácil del populismo. Deben asumir el desafío histórico en el que estamos, y aceptar el esfuerzo que supone tener que construir un discurso político que construya tipos de sociedad.

El discurso político debe fomentar decisiones no con criterios que apelen a percepciones de la ciudadanía y del "pueblo", sino criterios democráticos de justicia como la igualdad, el pluralismo, el civismo, la imparcialidad, como principales principios rectores. Decir, por ejemplo, como se está escuchando últimamente de forma pública que no se pueden dar derechos politicos a los inmigrantes porque los ciudadanos no quieren y que esta decisión puede provocar pérdida de votos es un argumento populista que expresa perfectamente lo que estoy diciendo. Ampararse en lo que piensa el pueblo para justificar la quietud política es populismo encubierto; que el político diga lo que las emociones del pueblo expresa, es populismo.

Todos sabemos que un político no debe guiarse por el interés del ciudadano a secas, sino que debe plantearse en muchas ocasiones por qué tiene el ciudadano dicho interés y cuáles son las politicas que se pueden hacer para evitar que lo tenga. Si no aceptamos esto, entonces justificamos la

ARBOR CLXXXI 713 MAYO-JUNIO [2005] 101-114 ISSN: 0210-1963 
telebasura que nos embrutece. Este argumento, en temas de inmigración, debe adquirir el carácter de un principio. ¿Qué vias seguir para diseñar un discurso político de la inmigración que tenga como límite evitar la argumentación populista? No basta con saber y decir que estamos ante un reto histórico sin precedentes en nuestra sociedad moderna, no basta la politica como retórica, sino que debemos adecuar nuestra forma de hacer política a esta convicción. Debemos hacer politica teniendo un marco de referencia generacional, recuperar la política como pedagogía, como responsabilidad. Ayudar al ciudadano a asumir esta nueva realidad y romper la lógica que actualmente prevalece en la mayoría de los espacios públicos de los barrios de uinmigrante-invasor/ciudadano-colonizado". La gestión de la inmigración demanda hacer política en el sentido histórico del término. El ciudadano lo está esperando. No contribuyamos en engrosar la desafección politica, especialmente en temas donde el populismo se nutre de los vacíos que generan las preguntas en torno a la inmigración.

\subsection{Vinculo entre dos procesos de multiculturalidad: la multinacionalidad y la inmigración}

Igual que la inmigración, la multinacionalidad es un proceso que se da en numerosos paises europeos (Bélgica, España, Gran Bretaña, Irlanda), por no mencionar Canadá y Québec como caso paradigmático. Estos dos procesos, que inicialmente se han analizado de forma separada, deben articularse. La forma en cómo se gestionará este vínculo es un asunto importante en España. Las demandas de acomodación de inmigrantes en concepciones de identidad nacional no estatal puede tener unos efectos sobre las demandas de autogobierno. El análisis de cómo estas dos demandas de acomodación interactúan está todavia por hacer. Por el momento, estamos en la fase más permeable a preguntas. Por ejemplo, ¿cómo los inmigrantes son percibidos por el proceso de construcción de identidad nacional no estatal?, ¿Debe considerarse la acomodación de los inmigrantes dentro de las identidades nacionales articuladas en niveles sub-estatales?

En España este debate apenas se ha introducido en la agenda política en Cataluña. Podemos incluso decir que si bien con el derecho al voto tenemos un ejemplo de tema existente en la agenda social pero no política, nos encontramos con este tema en una situación inversa: es un asunto que pertenece más a la agenda política, pero no a la agenda social. El principal desafio para los partidos nacionalistas es precisamente introducir el tema en el debate social. Por el momento, sólo han existido algunas intenciones de plantear esta perspectiva, pero no podemos decir que el tema se halla ni siquiera enfocado políticamente. Existe más en los pasillos parlamentarios que en el Pleno. Ahora bien, podemos comentar ya algunos puntos.

En primer lugar, se debe gestionar el vinculo entre dos categorias: la de las competencias y la de la identidad. Por el momento el tema se está planteando, como muchos otros, en términos de competencias y no de identidad. Esto significa que se están concentrando en las demandas de competencias para poder controlar los flujos de inmigrantes, con el argumento lícito (comprobado estadisticamente) de que la mayoría de los inmigrantes que entran en España se instalaran en Cataluña. Ahora bien, también se pueden profundizar otras demandas de carácter más de reconocimiento, como el hecho que los inmigrantes deban firmar la lealtad al rey para poder tener la nacionalidad española, o bien que se exija la lengua española, pero no se insista en las otras lenguas para poder acomodarse en territorios bilingües, 0 bien simplemente para optar a la nacionalidad. ¿Qué pasaria si un marroqui que hablase solamente gallego/euske$\mathrm{ra} / \mathrm{catalán} / \mathrm{se}$ negase hablar castellano en su lugar de trabajo? Estas son cuestiones prácticas que, aunque todavía no estén presentes en las discusiones, constituirán sin duda temas de disputa en un futuro próximo.

En segundo lugar, y como he apuntado en otras secciones, el debate se está enfocando en términos de identidad nacional (no estatal). En este punto plantearemos unas dudas. Si el movimiento nacionalista utiliza el discurso de la identidad, entonces estará utilizando una de las lógicas discursivas del populismo de derechas, el que construye sus argumentos para proteger la identidad nacional estatal. El discurso nacional-identitario puede provocar una etnización del proyecto mismo nacionalista, con consecuencias no previstas. En este marco, quizás deberia re-enfocarse el discurso en términos de acomodación de los portadores de la nueva multiculturalidad (los inmigrantes) con la multiculturalidad ya existente (la multinacionalidad). No deben construirse argumentos que enfrenten o perciban como antagónicos estos dos procesos. Estos dos procesos no necesariamente colisionan cuando coinciden en un mismo espacio territorial, sino que deben fusionarse. Esto significa que es legítimo pedir, o proporcionar instrumentos para ello, que los inmigrantes que quieran instalarse en Cataluña también hablen catalán. Pero en este caso también debemos reconocer que 
las personas "hablanla lengua de su lugar de trabajo». Las motivaciones que puede tener una persona de origen inmigrante para hablar catalán serán directamente proporcionales a dos factores: su intención de instalarse de forma definitiva en Cataluña, la necesidad de utilizar la lengua catalana para el trabajo, y poder ascender laboral y socialmente. Para fomentar estas motivaciones, los dos procesos deben poder vincularse entre sí. En este caso también es lógico tener la precaución de no utilizar la lengua no estatal en términos de finalidades: un inmigrante puede muy bien decir «hablo Español (catalán/euskera/gallego), pero no me siento integrado".

Recogiendo los argumentos de tres autores que han reflexionado sobre la relación entre inmigración y minoría nacional, como son J. Carens (1995, 2000 cap. 6), W. Kymlicka (2001) y R. Bauböck (2001), podemos tener una cartografía de temas básicos que conforman el debate actual. Los tres enfocan el tema como siendo de gestión de identidad y comparten siete premisas básicas. En primer lugar, que la acomodación de los inmigrantes supone una doble transformación: la identidad de los inmigrantes y la de nuestra sociedad. En segundo lugar, que todos los argumentos que se den no pueden vulnerar los valores liberales y democráticos. Es decir, que las reflexiones y producción de argumentos que se hagan tienen que tener como marco de referencia normativo de evaluación los valores liberales-democráticos. En tercer lugar, se trata de hablar de cómo se gestiona la identidad (primera premisa) sin sobrepasar los límites de los valores de nuestra tradición democrática liberal (segunda premisa) en el contexto de las diferentes esferas públicas (espacio de interacción entre las personas con las instituciones públicas). Se trata de discutir, pues, la vida pública de la persona y no privada. En este marco, una redefinición de qué significamos por cultura pública común en un contexto donde se solapan dos tipos de multiculturalidad (la de los inmigrantes y la de las naciones sin Estado) tiene el carácter de una exigencia, puesto que constituirá el principal marco de referencia para legitimar politicas. En cuarto lugar, comparten la concepción que las demandas de los inmigrantes y de las naciones minoritarias son en un principio conflictivas, puesto que los inmigrantes tenderán a integrarse en la cultura mayoritaria, con el consecuente efecto de convertirse en un elemento más de presión en el proceso de construcción nacional. De lo que se trata es de convertir este elemento inicial de presión en una ventaja que contribuya al propio desarrollo del proyecto nacional. Es decir, y en nuestros términos, de pasar de una forma re-activa de percibir el tema en una forma pro-activa, dónde la inmigración se convierta en una fuente necesaria para el propio desarrollo nacional. Los tres autores comparten que esto no solamente es posible, sino que es necesario y que está de acuerdo (no vulnera) con los principios de la democracia y del liberalismo. En quinto lugar está la premisa que si bien existe un vínculo directo entre las políticas de inmigración y la comprensión de la comunidad política, esta relación adquiere un carácter vital para comunidades culturales minoritarias. Es un hecho que la respuesta que da la sociedad a la inmigración forma parte de su auto-comprensión como sociedad (Carens, 1995; 20). La inmigración tiene un efecto espejo en todos los niveles, desde el individual hasta el social (R. Zapata-Barrero, 2002). Por lo tanto las políticas que se hagan afectan directamente la auto-comprensión como comunidad política minoritaria. En este marco, esta auto-comprensión solamente puede adquirir una expresión política si existe una forma propia de autogobierno. Sin autogobierno no es posible mantener y desarrollar la comprensión que una comunidad politica tiene de ella misma. Por lo tanto, al hablar de gestión de inmigración para naciones minoritarias estamos frente a uno de los fundamentos que legitiman las demandas de autogobierno. Como sexta premisa, el esfuerzo argumentativo de los autores quiere expresar la necesidad de concebir las demandas de los inmigrantes y las del autogobierno como compatibles y no como mutuamente excluyentes, de tener una concepción inclusiva y convergente. En este caso, el núcleo de la reflexión es si la nación minoritaria es capaz de ser ella misma multicultural (Kymlicka, 2001; 278), incluyendo dentro de su autoconcepción la multiculturalidad que expresa la presencia de inmigrantes, y que esta multiculturalidad, siguiendo la premisa anterior, contribuya al desarrollo de su comunidad.

Por último, pero no menos importante, como séptima premisa destacamos que una de las nociones que sirve a los tres autores para describir la situación no es tanto la de desigualdad sino la de desventaja. Especialmente Carens y Bauböck la utilizan reiteradamente en sus argumentaciones. Es importante circunscribir bien el significado de esta noción como recurso analítico por precisar los temas que nos ocupan. Si bien aceptamos que tanto los inmigrantes como la minoría nacional están en situación de desventaja y aceptamos que de hecho esta es la situación inicial que está a la base de la reflexión misma que queremos hacer, de lo que se trata ahora es de comparar las dos situaciones para saber si estamos utilizando una misma noción pero para describir referentes diferentes. La situación de desventaja para los 
inmigrantes y para las naciones minoritarias se producen por razones diferentes. De lo que se trata es de saber también si esta situación se empeora o no al vincularse los dos, La política de inmigración del Estado empeora la situación de las naciones minoritarias en cuanto que no le proporciona herramientas ni recursos para que ella misma gestione este proceso que afecta su propio desarrollo como comunidad cultural. Las líneas de reflexión que se hagan tienen que tener como restricción política enfocar el tema de tal manera que el vínculo entre inmigración y autogobierno no empeore la situación de los dos. Que la mejore seria lo ideal, pero creo que es razonable mantener que al menos no lo empeore. Estamos, pues, ante un tema que tiene como fuente prioritaria de argumentación las políticas de autogobierno, es decir, aquellas políticas que tienen como última base de legitimación la gestión de la propia comunidad cultural minoritaria. En este sentido, toda política de inmigración gestiona en última instancia la pertenencia de los inmigrantes a una comunidad política. Aqui estarian en los enfoques clásicos. De lo que se trata es de plantearse este tema pero en sociedades dónde existe una doble pertenencia: la de la comunidad política mayoritaria estatal, y la de la comunidad expresada por la nación minoritaria. Es a partir de esta base que se justifican las acciones. Veamos ahora el tercer gran tema.

\subsection{Secularismo o la gestión del pluralismo religioso}

Todos reconoceremos, y muchos lo tomarán como los únicos aspectos a tener en cuenta, los casos de los oratorios y de las mezquitas para la población islámica, pero también casos de enfrentamiento (zonas de conflicto) para no dejar que unas identidades religiosas tengan un espacio público. Me refiero a los casos de los velos, que también se ha dado en numerosos paises europeos, siendo Francia el caso más ilustrativo. También recientemente se están dando nuevos casos en Francia que entrarian dentro de esta categoria, como el establecimiento de horarios en piscinas municipales para niñas musulmanas. Estas situaciones son muy diferentes entre sí, pero todos coinciden en que se trata de que unas zonas de contacto entre dos tradiciones religiosas (la católica y la islámica) se convierten en zonas de conflicto. Gestionar estas zonas se convierten en un desafío vital para la propia madurez democrática de nuestras sociedades.

El enfoque dado a estos casos sobre la pretendida secularización de nuestra tradición, o del laicismo de nues- tras sociedades, es simplemente pura hipocresía. Nuestras estructuras han estado pensadas en un marco religioso de tradición cristiana. Por lo tanto, aventaja más a una religión que a otra. De lo que se trata no es de islamizar el espacio público, sino el de gestionar el pluralismo religioso en términos de igualdad (sin discriminación). Estos casos siempre han acabado resolviéndose aventajando una religión frente a otra si se hubiera encontrado con problemas similares, y vulnerando derechos democráticos tan básicos como es el de la libertad de culto. Asimismo, el criterio de la religión cristiana es uno de los que se suelen utilizar como criterio de selección de inmigrantes en el momento de establecer la admisión dentro de nuestras fronteras.

Es cierto que la percepción social de la inmigración generalmente va asociada a los musulmanes, como minoria religiosa. En base a este vínculo público se construyen unos discursos conservadores sobre la identidad europea y la civilización europea que apela a las tradiciones cristianas y construye el Islam como nuevo pueblo bárbaro. España está ya participando en esta dinámica.

Este tipo de discurso tiende, asimismo, a confundir religión y cultura. El resultado de esta confusión contribuye a crear una concepción negativa del proceso de multiculturalidad. De este modo, crea una concepción religiosa de la cultura en lugar de fomentar lo contrario, una concepción cultural de la religión. En el fondo, se trata de saber si España (y Europa) es capaz de gestionar la población de origen musulmán creando zonas de contacto sin que acaben siendo zonas de conflicto. Se trata, en el fondo, de saber si Europa es capaz de considerar a los musulmanes como los Hispanos para los Estados Unidos. La población más numerosa de inmigrantes que plantean temas identitarios culturales no necesariamente religiosos. Los efectos espejo que provoca son claros, si el velo se prohibe, también deberian prohibirse los collares con cruces, los piercing, y todos los signos que denoten de una forma directa identidades grupales (F. Colom, 1998; J. de Lucas, 2003). Qué vuelvan los uniformes en las escuelas (?!). El tratamiento imparcial de estos temas es uno de los enfoques a tener en cuenta para gestionar el proceso de multiculturalidad. ${ }^{4}$ Esta regla de la imparcialidad nos dice que los principios que legitiman una politica deben poder ser aceptados por todos, los inmigrantes y los ciudadanos. La gestión del pluralismo religioso es una politica que debe incluir "todos los puntos de vista posibles". 
En España este debate debe ir también acompañado de una discusión profunda sobre el proceso de construcción de nuestra tradición y la identidad española, básicamente formada como identidad reactiva contra la identidad musulmana. Este argumento tiene un carácter práctico evidente. En efecto, en España, siempre que los miembros de una comunidad musulmana deseen construir una mezquita en una ciudad, una reacción profundamente arraigada e inmediata de protesta vecinal comienza, apoyado generalmente, o no reprimido por lo menos, por autoridades locales. La actitud de autoridades locales hacia la comunidad musulmana se puede resumir por su reconocimiento de la existencia de un lugar de culto en los hogares privados, centros de comunidad, etc. y su repugnancia para reconocer la necesidad de dar a musulmanes la visibilidad pública que sí tiene la iglesia católica (J. Moreras, 2003). La gestión de esta primera pregunta constituye casi siempre una señal para las autoridades políticas y los ciudadanos al analizar la manera como España está gestionando la presencia musulmana. Invariablemente, las encuestas de la opinión pública sobre estos asuntos revelan que la mayoria de ciudadanos españoles liga su oposición a los inmigrantes en general, a la comunidad musulmana en particular, especialmente la más numerosa, la comunidad marroqui. ${ }^{5}$ Es un hecho que el tema de los musulmanes ha aparecido en la esfera pública las imágenes algo rígidas unidas a ellas. Casi todas las noticias negativas de la inmigración se relacionan con la comunidad musulmana.

Es también una realidad que la identidad española, como la europea pero con muchos más puntos históricos de referencia, se ha construido en oposición a lo musulmán engeneral y lo marroqui en concreto, considerado en términos pejorativos como «el Moron. Nadie puede negar que la tradición española no se puede entender sin la herencia islámica y el patrimonio cultural. ¿Cómo España gestiona el hecho de que una imagen estereotipada histórica del Moro gobierna la opinión pública y las discusiones políticas? ¿Cómo puede España luchar contra esta opinión negativa profundamente arraigada sin amenazar su identidad nacional? ¿Cómo pueden los musulmanes residentes en España expresar su auto-identidad en una sociedad y en un entorno político que lo rechaza?, ¿Debemos asumir que el Islam pertenece "a una anomalia histórica»? Cualquiera que sean las respuestas, el debate se centra en la relación entre la presencia de la identidad comunitaria musulmana y la tradición de la identidad española. ${ }^{6}$ Por el momento el debate puede seguir dos lógicas: Una primera que dice que el Islam es una anomalia dentro del proceso histórico español (¿una anormalidad de ocho siglos?), algo extraño a nuestra tradición e identidad culturales. La segunda lógica es la que establece que el Islam pertenece a nuestra tradición e identidad culturales. Dependiendo de la lógica que uno siga tendrá una fuente diferente de producción de discursos políticos. El primer enfoque tiende a considerar dos tradiciones separadas que han estado históricamente en desacuerdo. Tenderá a seguir un discurso político que enfatize la diferencia entre las tradiciones musulmanas y españolas, qué separa las dos tradiciones. El segundo enfoque defenderá que hay una tradición (en lugar de dos) que ha convergido históricamente. Esta línea da lugar a un discurso político que acentúa lo común de las dos tradiciones (M. A. Roque, ed., 2003).

El Islam en España no es una nueva realidad que los ciudadanos están descubriendo, sino un hecho histórico que la gente tiende para reprimir, en términos casi freudianos. En este marco la pregunta directa importante es la siguiente: ¿Es posible incorporar la herencia musulmana en la tradición de la ciudadanía española sin amenazar la identidad española? Dejo aquí la cuestión para el debate y prosigo mi linea de argumentación con el quinto tema pendiente de un discurso político.

\section{El marco de la UE: la propuesta de ciudadanía cívica de la Comisión Europea como vía para la gestión de la multiculturalidad}

En el marco de Tampere, la Comisión introdujo en noviembre de 2000 la categoría de ciudadanía cívica para conceptuar la figura del inmigrante residente permanente o lo que en el debate académico se suele denominar como denizenship europeo (T. Hammar, 1990 y 2003). Con esta propuesta se hace un cambio cualitativo importante, puesto que esta noción se transforma en principal eje de las políticas de integración de la UE, especialmente desde las conclusiones de la presidencia del consejo europeo de Salónica (19-20 de junio de 2003) y las conclusiones de Tampere de junio de $2004{ }^{7}$ donde se reconoce por primera vez la necesidad de elaborar una política comprensiva y multidimensional de integración para los nacionales de terceros estados legalmente residentes en uno de los Estados Miembros (A. Solanes, 2004). Este proceso de europeización de una categoria de inmigrante está también vinculada a la Directiva del Consejo, relativa al estatuto de los nacionales de terceros paises residentes de larga duración. ${ }^{8}$ Este nuevo frente que lidera la Comisión es importante, no solo por el enfoque 
que propone, sino por la "filosofía" que establece. De lo que se trata ahora es de introducir en el vocabulario de la UE el concepto de integración aplicado a los inmigrantes. Para ello, es necesario antes tener una categoría europea de inmigrante: el del residente permanente bajo el concepto de ciudadanía cívica Una nueva etapa comienza. Por primera vez la lógica de los inmigrantes entra en el proyecto común europeo. Este debate interesa interpretarlo teniendo en cuenta los indicadores de multiculturalidad que hemos introducido de España. Desde la realidad española, este debate tiene dos posibles interpretaciones: considerar la ciudadania cívica como una propuesta de política innovadora de inclusión o bien considerar esta propuesta como formando parte de la tradición de una política clásica de adquisición de ciudadania, siguiendo pautas estatales.

Teniendo en cuenta el proceso de cambio de paradigma donde nos encontramos como enfoque (cap. 1) y los indicadores de multiculturalidad como marco de reflexión (cap. 2) mi propósito es destacar cómo este debate reproduce una nueva fractura dentro de la UE entre una política más abierta (innovación) y más cerrada (tradición) de inmigración. Al final haré un balance final trasladando este dilema entre tradición e innovación en el seno del nuevo marco de políticas de inmigración: el programa de la Haya, iniciado en noviembre de 2004.

\section{1 ¿Nueva filosofía de la UE? Dos modelos de ciudadanía cívica: tradición o innovación}

La iniciativa de la Comisión de conceptuar al inmigrante residente permanente (denizen europeo) es un paso adelante $\sin$ precedentes. Los discursos que ha provocado son de por si también un avance en tanto que el tema se incorpora a la agenda europea y ayuda a socializar una cuestión de importancia vital para el propio futuro de la UE.

Supone un paso importante por, al menos, tres razones:

1. Incluye por primera vez la lógica del inmigrante dentro de los debates de construcción de la UE. 0 invirtiendo el argumento, la UE ya no es solo asunto de nacionales europeos sino también de nacionales de terceros paises de larga duración o ciudadanos cívicos.

2. Produce un salto cualitativo al cambiar la percepción de la UE: se pasa de una percepción de la inmigración como exclusivamente de control de flujos y asuntos de seguridad, a una basada en la integración. Esto significa que por vez primera se introduce en el vocabulario europeo el término de integración, inicialmente destinado a describir el proceso de construcción de la UE. Ahora 'integración' tiene un nuevo referente: el inmigrante. Con esta nueva noción en los círculos europeos por primera vez la lógica de los inmigrantes entra en el proyecto común europeo. ${ }^{9}$

3. Es una noción mucha más extensa, inclusiva, que la clásica de ciudadanía europea estrictamente centrada a los nacionales de Estados. Por lo tanto permite conceptuar a una población que quedaba desatendida con la noción exclusivamente estatal y vinculada a la nacionalidad (de un Estado Miembro) de ciudadanía europea. Esta extensión se efectuá con el establecimiento de un sistema de derechos y deberes comunes entre nacionales europeos y de terceros paises.

Aunque existan motivos para ello, no comparto el optimismo (sin condiciones) de J. de Lucas (2004), quien interpreta este paso importante en el camino hacia la obtención de un estatuto de residente europeo para los inmigrantes que, cumplidas determinadas condiciones, puedan ser equiparados en sentido estricto, es decir, en términos de igualdad de derechos, a los ciudadanos europeos. Se trata de la recuperación de la idea de que ciudadano es el que habita en la ciudad, no sólo el que nace en ella. Si bien comparto con el profesor De Lucas que esta integración cívica es ante todo una garantía de integración política y jurídica, esto es, garantizar a los inmigrantes una igualdad jurídica y política, y, como consecuencia, garantizar el acceso de los inmigrantes a la ciudadanía y a sus derechos, ${ }^{10}$ discrepo en tanto que interpreta todo el debate como si se tratara de un único objeto: la ciudadanía civica. Creo que estamos en presencia de dos modelos muy diferenciados, y que sólo uno ha sido recogido por la Comisión y la Constitución. El modelo del que habla Javier de Lucas ha sido, simplemente, ignorado.

En estos debates, vemos que se perfilan dos concepciones muy diferentes: una concepción tradicional que sigue una lógica nacional defendida particularmente por la Comisión y de una forma menos explícita, pero también tácita, por el Parlamento, y una concepción innovadora, que sigue una lógica postnacional defendida principalmente por el Comité de las Regiones y el Comité Económico y Social Europeo. En la primera, la introducción de la noción de ciudadanía civica responde a intenciones de armonizar el estatuto de la residencia permanente para que adquiera una 
dimensión europea, y como paso previo para la adquisición de la ciudadanía nacional, en manos de los Estados. De esta forma se soluciona el tema de la asimetria que existe actualmente en la adquisición de la ciudadanía europea, puesto que cada Estado regula de forma diferente la «ciudadania permanenten, como criterio para la adquisición de la nacionalidad (ciudadanía) de un Estado (R. Zapata-Barrero, 2003). Pero se sigue defendiendo un vínculo: la concesión de la ciudadanía europea y de los derechos políticos para los inmigrantes queda todavía en manos de los Estados, quienes siguen vinculando acceso a los derechos políticos con la adquisición de la nacionalidad, y por lo tanto, sigue defendiendo una percepción nacional de la ciudadanía. En el otro extremo está una concepción de la ciudadanía vinculada a la residencia y no a la nacionalidad. Seria la residencia la que otorgaría el estatuto de la ciudadanía europea, sin necesidad de estar vinculada a la nacionalidad. En este nivel es donde seguiriamos la interpretación de De Lucas, donde la lógica europea seguiria más una lógica local (basada en el empadronamiento) y no estatal de la ciudadanía. Ésta es la que defienden tanto el CESE como el Comité de las Regiones. Entre ambas concepciones sólo una es la que ha llegado al Consejo Europeo y a la Constitución Europea: la ciudadanía sigue estando vinculada a la nacionalidad.

Ahora bien, sea cual sea la vía de interpretación de la ciudadanía cívica que adopte la UE, Tradición versus Innovación, queda un núcleo común que bien puede servir de un premisa para orientar el debate académico. Podemos resumir este núcleo del concepto de ciudadanía civica de la forma siguiente (me baso en R. Bauböck, 2004):

1. Es un estatus de derechos y deberes, similar a denizenship europeo dirigido a los residentes legales de larga duración. Esto significa que es un estatus paralelo al estatus de ciudadanía europea. Los nacionales europeos no adquieren el estatus de ciudadania cívica.

2. Si los beneficiarios del estatus son los residentes de larga duración de terceros paises, La residencia se convierte en criterio básico para el acceso a la ciudadanía cívica.

3. El área del estatus es todo el territorio de la UE, independientemente del estado. Esto significa que todo residente permanente podrá gozar del mismo estatus independientemente del Estado donde resida.

4. La base de este estatus es la igualdad juridica (igualdad de trato) y la no-discriminación por razón de nacionalidad.

5. Los principales derechos que otorga son los de la Carta de Derechos Fundamentales.
6. Incluye derechos básicos como: derecho de residir, recibir educación, trabajar sin discriminación respecto a ciudadanos de la Unión

7. Contempla la extensión de derechos locales de voto.

8. Considerado como primera etapa para recibir nacionalidad de un Estado, por tanto la ciudadanía europea.

Vemos que existen puntos, dentro de este núcleo común, que llevan hacia la tradición (especialmente el punto 8), mientras que otros, también presentes en el concepto, que siguen más la Innovación (especialmente el punto 3). Esta será la disyuntiva que deberá resolverse en el nuevo marco de discusión de la inmigración: el programa de la Haya.

\subsection{Proceso de innovación conceptual en un marco tradicional estatal: el marco de La Haya}

Estamos en fase de la UE donde se mezclan procesos de innovación conceptual (ciudadanía civica) con nociones clásicas que por vez primera entran en el seno del debate institucional de la UE (integración). Lo que queda claro es que la noción de integración que habitualmente pertenecía al vocabulario europeo para significar el proceso de construcción europea, ahora se le ha dotado de un nuevo referente empirico: ciudadanía cívica.

Acaba de finalizar una primera etapa en el marco de Tampere (1999-2004), donde se inicia formalmente el proceso de construcción de un enfoque europeo de la inmigración y que ha desembocado en dos directivas de suma importancia: la referente a la reagrupación familiar (Directiva 2003/86/CE, sept. 2003), y la relativa al estatuto de los nacionales de terceros países residentes de larga duración (Directiva 2003/109/CE, nov. 2003). Entramos ahora en un nuevo periodo iniciado tras las conclusiones de la presidencia del consejo europeo de Salónica (19-20 de junio de 2003) que ha abierto un nuevo marco para discutir la gestión de la inmigración en la UE: el programa de la Haya. Cinco años después del Consejo Europeo de Tampere, cuando acordó un programa que sentaba las bases para importantes logros en el espacio de libertad, seguridad y justicia, este nuevo programa tienen como objetivo avanzar sobre dichos logros y hacer frente con eficacia a los nuevos retos. Este nuevo programa plurianual para los próximos cinco años refleja las ambiciones expresadas en el Tratado por el que se establece una Constitución para Europa y tiene en cuenta la evaluación de la Comisión que el Consejo Europeo de junio de 2004 acogió con satisfacción, así como la Recomendación sobre

ARBOR CLXXXI 713 MAYO-JUNIO [2005] 101-114 ISSN: 0210-1963 
la utilización de la votación por mayoría cualificada y el procedimiento de codecisión aprobada por el Parlamento Europeo el 14 de octubre de 2004. El Programa de La Haya trata todos los aspectos de las políticas relativas al espacio de libertad, seguridad y justicia. En lo que respecta a la ciudadanía e inmigración, trata concretamente los derechos fundamentales y la ciudadanía, el asilo y la migración, la gestión de las fronteras, la integración.

En relación con la integración de los nacionales de terceros paises, el programa vincula la integración con la estabilidad y la cohesión en nuestras sociedades (apartado 1.5). Uno de los objetivos básicos que traza es la prevención del aislamiento de ciertos grupos. Para ello, defiende un planteamiento integral que implique a los interesados al nivel local, regional, nacional y de la UE y seguir la igualdad de oportunidades con la finalidad de participar plenamente en la sociedad. El principio de coordinación con las políticas nacionales de integración y las iniciativas de la UE en este ámbito resulta uno de los ejes centrales. A este respecto, expresa la necesidad de establecer los principios de base comunes que sirvan de fundamento para un marco europeo coherente de integración. Dichos principios, que habrán de conectar todas las áreas políticas relacionadas con la integración, deben incluir por lo menos los siguientes aspectos de la definición de Integración (recogiendo parte de los debates entre instituciones desde la Comunicación de la Comisión del 2000):

- Es un proceso continuo y bidireccional que implica tanto a los ciudadanos de terceros países con residencia legal como a la sociedad de acogida

- Incluye la política contra la discriminación

- Implica el respeto por los valores básicos de la Unión Europea y los derechos humanos fundamentales

- Requiere unas capacidades básicas para la participación en la sociedad

- Se basa en la interacción frecuente y el diálogo intercultural entre todos los miembros de la sociedad, en el marco de foros y actividades comunes encaminados a mejorar la comprensión reciproca

- Se extiende a una variedad de áreas políticas, incluidos el empleo y la educación.

La intención de la UE es que este marco basado en estos principios comunes formará los cimientos para iniciativas futuras, basándose en objetivos y medios de la evaluación claros. El Consejo Europeo invita a los Estados miembros, al Consejo y a la Comisión a que promuevan el intercambio estructural de experiencias e información sobre la integración.
Siguiendo los objetivos de Salónica y en este marco se sitúa también el Handbook on integration elaborado por el Migration Policy Group a petición de la Comisión Europea (dirección de Justicia, Libertad y Seguridad). En efecto, uno de los objetivos de Salónica es desarrollar la cooperación y el intercambio de información sobre integración para promover iniciativas politicas. Este Manual constituye una de sus primeras acciones. Su pertinencia cubre una doble necesidad: por un lado, hace un repaso de las principales acciones que se están poniendo en práctica en diferentes países (es un catálogo de buenas prácticas, de ideas para inspirar nuevas iniciativas en materia de integración); por otro lado, esta destinado a un tipo de actor muchas veces olvidado pero que (todos sabemos) tiene una gran responsabilidad llevando la mayoria del peso social que implica la gestión de la inmigración: a los responsables de elaborar politicas y formular objetivos, y a los profesionales y técnicos que diseñan las acciones. Este colectivo está desatendido políticamente, y es nuclear para el éxito de la gestión de la acomodación de los inmigrantes y ciudadanos.

La estructura del manual nos da ya muchas indicaciones de su filosofía: trata de programas de acogida (cap. 1), de la participación política y políticas de ciudadanía (cap. 2) y de indicadores necesarios para evaluar programas y comparar paises (cap. 3). Luego, en un anexo presenta un plan para elaborar programas, cuya primera recomendación, que tiene el estatuto de fundamento, es la necesidad de crear una complicidad y compromiso de todos lo actores implicados (stakeholders) (sindicatos, asociaciones de inmigrantes, confesiones religiosas, empresarios, ong, etc). Recogiendo gran parte de los debates entre instituciones, en este manual se define la integración como un proceso bidireccional que centra la atención en la actitud de les sociedades receptoras, en su ciudadanía y sus estructuras institucionales; se insiste en que existe un vínculo entre participación politica y sentimiento de pertenencia; se recuerda que un buen plan de acogida debe tener un enfoque de formación de la ciudadania (lingüistica y civica); que todo plan debe centrarse en las capacidades y formación del inmigrante recién llegado para que pueda tener las mismas oportunidades en la sociedad; sigue el sentido común de conceder derechos políticos, al menos a nivel local y para residentes de larga duración, y de muchas más acciones e ideas. Tenemos, pues, elementos para ser optimistas, puesto que ya que se ha empezado no sólo a hablar de integración y de búsqueda de normas e indicadores comunes de interacción, sino también se ha abierto un debate sobre un nuevo concepto de ciudadanía que incluya a los inmigrantes, y por lo tanto, 
que permita a los inmigrantes considerar por vez primera que la UE les concierne (R. Zapata-Barrero, 2003).

Todos los debates entre instituciones que hemos trazado y que se enmarcan dentro de la lógica de Tampere de introducir y desarrollar un espacio de Liberad, Seguridad y Justicia (la Santisima Trinidad de la UE) lleva a la necesidad de considerar a los inmigrantes residentes permanentes como sujetos también de este espacio, y no como excluidos hasta ahora. En este nuevo escenario, el concepto de ciudadanía cívica daría a los inmigrantes ciertos derechos y obligaciones, incluido el derecho a vivir y trabajar en otro Estado miembro de la UE. Estos derechos se adquiririan al cabo de unos años con la intención de equipararlos con los derechos que gozan los ciudadanos de la UE (Comisión Europea, Libertad, seguridady justicia para todos, Europa en Movimiento, 2004; 9).

La Comisión no sólo ha conseguido introducir un nuevo concepto dentro del vocabulario europeo: el de ciudadanía cívi$c a$, sino que desde entonces lo utiliza de forma sustantiva para hacer sus comunicaciones, siempre relacionados con su discurso (en construcción) de la integración. Por ejemplo al hablar de la integración en la sociedad de acogida, asevera: ${ }^{11}$

"Si, dado el carácter forzoso de la migración de las personas que se benefician de la protección internacional y las necesidades específicas debidas a su condición de exilados, deben tenerse en cuenta los derechos incluidos en el Convenio de Ginebra, los retos de la integración son los mismos en términos generales que para los otros emigrantes procedentes de terceros paises así como para las sociedades de acogida en relación con las cuestiones de la nacionalidad, la ciudadania cívica y el respeto de la diversidad, asi como la política social y de acceso al mercado laboral, la educación y la formación.»

Hemos visto que inevitablemente los argumentos que se dan forman parte de desiderata y no estrictamente de facto, aunque debemos valorar, valga el rodeo que hago, que el facto es que se expresen estos desiderata.

Con estos debates se abre una triple categoría de población en la UE: los inmigrantes (los que no son de larga duración, menos de cinco años según la Directiva citada), para quienes todavia no se pueden beneficiar ni del territorio ni de los derechos que otorga la Constitución Europea, los ciudadanos civicos (los inmigrantes residentes de larga duración, nacionales de terceros paises o denizens europeos), quienes pueden beneficiarse de la Carta de Derechos fundamentales, y los ciudadanos europeos o nacionales de Estados Miembros.
Desde el punto de vista de construcción de una ciudadanía que tenga en cuenta la realidad multicultural en Europa, de construcción de un enfoque europeo de Ciudadanía Multicultural (T. Modood, et. al., eds 2005), reconozco que tengo una interpretación ambivalente. Con el análisis del debate entre instituciones sobre la ciudadanía cívica y su ubicación dentro de los grandes marcos de referencia discursivas politicas de la UE (Tampere, Salónica, La Haya) podemos ver la botella media vacía (Tradición) o media llena (Innovación) Déjenme formular los principales argumentos para acabar:

\section{Media llena: Se ha construido un criterio objetivo... (Innovación)}

Lo que supone es una armonización de la categoría de residente permanente o denizenship de la Unión. Sólo es el resultado de un proceso de armonización, pero no un concepto cualitativo nuevo. Se institucionaliza una categoría que ya existe en los Estados: la de residente permanente. $Y$ se le otorga a los residentes permanentes la igualdad jurídica y de trato.

\section{Media vacía:... pero a imagen estatal (Tradición)}

- Es una categoría que sirve de criterio a la UE para armonizar el código de nacionalidad, y evitar, así, la situación asimétrica que todavia existe en la UE en criterios de otorgar nacionalidad y por tanto, como la ciudadanía es una categoría derivada, la ciudadanía europea (R. ZapataBarrero, 2003d)

- La ciudadanía europea no se ve para nada afectada, sigue siendo: superpuesta o de segundo grado, condición juridica y derivada en tanto que su determinación es competencia exclusiva de los EEMM.

- Lo que se está haciendo es otorgar categoría europea a los nacionales de los terceros estados, puesto que se necesita antes construir el sujeto antes de hacer políticas

- Lo que se está haciendo es construir una categoría de residente permanente a imagen estatal, sin nada de innovador ni cambio cualitativo. El cambio revolucionario seria que la ciudadanía civica sea el criterio para la ciudadanía europea, $\sin$ necesidad de depender de criterios de acceso a la nacionalidad de un Estado Miembro.

En resumidas cuentas, con la noción de ciudadania civica existe una nueva filosofía de la UE que busca incluir a los inmigrantes residentes como sujetos de derecho y sujetos

ARBOR CLXXXI 713 MAYO-JUNIO [2005] 101-114 ISSN: 0210-1963 
políticos de la misma UE, y no sujetos derivados mediados por la definición que den los Estados Miembros (EEMM) (A. Gerber, 2004). Esta nueva filosofía tiene el carácter de una salto cualitativo. La cuestión ahora es saber si este cambio de enfoque es un cambio conservador o progresista, en el sentido que mantiene una lógica tradicional estatal de percepción o bien no. El argumento que he defendido en este articulo es que ambas direcciones son hoy en día posibles, y que el futuro marco del Programa de la Hague tiene como nuevo reto precisamente la de definir el camino trazado hacia una $u$ otra dirección (Conclusiones de la Presidencia-Bruselas, 4 y 5 de noviembre de 2004).

\section{NOTAS}

* Este artículo se basa en aportaciones de dos conferencias: "Bases para interpretar la multiculturalidad en España como paso previo para un Pacto de Estado", conferencia realizada en el seminario "Inmigración y Sociedad" de la Fundación Pablo Iglesias (Madrid, 25-26 de noviembre 2004) y una ponencia presentada en el I/ Seminario; Inmigración y Europa: cinco años después de Tampere de la Fundación Cidob (Barcelona: -17 de diciembre 2004). Este capitulo forma parte de un proyecto de investigación del Ministerio de Educación y Ciencia titulado: La politización de la inmigración: relación entre el discurso político y social en España y políticas de la UE en torno a la inmigración (SEJ2004-04775/CPOL).

1 Comienza a existir una literatura sólida sobre cada uno de estos debates. Véanse entre la más reciente y alguna ya clásica de los noventa: T. A. Aleinikoff y D. Klusmeyer (eds. 2001), Análes de la Cátedra Francisco Suárez (2003), Y. Soysal (1994), R. Bauböck (1994), R. Bauböck y J. Rundell (eds. 1998), S. Castles y A. Davidson (2000), R. Koopmans y P. Statham (eds. 2000), W. AA. (2002), C. Joppke y M. Morawska (eds. 2003) J. de Lucas (2003).

2 Soy consciente que todas ellas requeririan más argumentos de justificación, pero no es propósito de este articulo elaborar un discurso demasiado hermenéutico, sino dejar por supuesto esta necesidad y aplicar estas premisas para interpretar el proceso en España, teniendo en cuenta el marco de la UE.

3 Existe una gran literatura empírica y teórica sobre estos partidos antiimmigrantes, de extrema derecha o nuevos populismos. Entre los más recientes, veánse: $P$. Taggard (2000), P. Perrinau (2001), Y. Mény y Y. Surel (eds. 2003), 0. Ihl et al. (2003), H. Betz (2004), P. A. Taguieff (dir. 2004).
4 Veremos más adelante este concepto de imparcialidad, basado en la noción de Th. Nagel (1991).

5 Véase un reciente libro que resume algunos estudios sobre este tema en V. Pérez-Diaz et al., (2004).

6 Éste es el principal argumento de R. Zapata-Barrero (2005).

$7 \operatorname{COM}(2004) 401$ final, de 2 junio, Comunicación de la Comisión al Consejo y al Parlamento europeo: Espacio de Libertad, Seguridad y Justicia: balance del programa de Tampere y futuras orientaciones.

8 Directiva 2003/109/CE del Consejo, de 25 de noviembre 2003, relativa al estatuto de los nacionales de terceros paises residentes de larga duración.

9 Véase al respeto uno de los primeros estudios que se sitúan en este marco en R. Süssmuth y W. Widenfeld (eds. 2004).

10 Véase J. de Lucas (2004), y también unas primeras reflexiones en J. de Lucas (2005).

11 Sección 2.2. de COM (2003) 152 final Comunicación de la Comisión al Consejo y al Parlamento Europeo sobre la política común de asilo y el programa de protección (Segundo informe de la Comisión sobre la aplicación de la Comunicación COM (2000) 755 final de 22 de noviembre de 2000).

\section{BIBLIOGRAFÍA}

Aleinikoff, T. A. y Klusmeyer, D. (eds.) (2001) Citizenship Today, Washington: Cornegie.

Anales de la Cátedra Francisco Suárez (2003) Ciudadanía e Inmigración (monográfico especial), n. 37.

Aubarell, G. y Zapata, R. (eds.) (2003) Inmigración y procesos de cambio: Europa y Mediterráneo en un contexto global, Barcelona: Icaria.

Bauböck, R (2001) "Cultural citizenship, minority rights, and selfgovernment", T. A. Aleinikoff i D. Klusmeyer (eds.) Citizenship Today, Washington: Cornegie; 319-345.

Bauböck, R. y Rundell J. (eds) (1998) Blurred boudaries: Migration, Ethnicity, Citizenship.s, Ashgate Aldershot.

Bauböck, R. (1994), Transnational citizenship: membership and rights in international migration, Aldershot: Edward Elgar.

Bauböck, R. (2004) "Civic citizenship-A New Concept for the New Europen, R. Süssmuth y W. Weidenfeld (eds.); 146-163.

Betz, H. (2004) La droite populiste en Europe: extrême et democrate? Paris: CEVIPOF.

Carens, J (1995), "Immigration, Political Community, and the Transformation of Identity: Quebec's Immigration Policies in Critical Perspective", Carens, J (ed) Is Quebec Nationalism Just? Montreal: McGill-Queen's University Press; 20-81.

Carens, J. (2000) Culture, citizenship, and community, New York: Oxford University Press.

Castles, S. y Davidson, A. (2000) Citizenship and Migration, Londres: MacMillan.

Colom, F. (1998) Razones de identidad. Pluralismo cultural e integración política, Barcelona: Anthropos. 
Gerber, A. (2004) "'Civic citizenship' et multinationalitésn, Terra Incognita, 4; 72-73.

Hammar Tomas (2003) «Denizen and Denizenship", in: Atsushi Kondo and Charles Westin (eds.) New Concepts of Citizenship, CEIFO Publications, No: 93, Stockholm University.

Hammar, Tomas (1990) Democracy and the Nation-State. Aliens, Denizens and Citizens in a World of International Migration, Aldershot, Avebury.

Ihl, O. et al. (2003) La tentation populiste au coeur de l'Europe, Paris: La Découverte.

Joppke, C. y Morawska, M. (eds.) (2003) Toward assimilation and citizenship. Immigrants in liberal nation-states, Houndmills: Palgrave Macmillan.

KoopmansS, R. y Statham, P. (eds.) (2000) Challenging Immigration and ethnic relations politics, Oxford University Press.

Kymlicka, W. (2001), «Minority Nationalism and Immigrant Integration», Will Kymlicka, Politics in the Vernacular , Oxford: 0xford University Press.

Lucas, J. de (2003) Globalizacion e identidades, Barcelona: Icaria.

Lucas, J. de (ed) (1999) Los derechos de las minorias en una sociedad multicultural, Madrid: C.G.P.J.

Lucas, J. de (2005) "Un modelo alternativo de ciudadania e inmigración" en el Seminario Inmigración y Europa, España en la construcción de una política europea de inmigración, Barcelona: Fundación Cidob; 115-127.

Lucas, J. de (2004) «El derecho frente al fenómeno de las nuevas migraciones", Manuscrito presentado en seminario sobre inmigración y sociedad, Fundación Pablo Iglesias, Madrid 25-26 noviembre.

Meny, Y. y Surel, Y. (2000) "Introduction", in Par le people, pour le people: le populisme et les démocraties, Paris: Fayard; 9-35.

Migration Policy Group (J. Niessen y Y. Schiebel) Handbook on integration for policy makers and practicioners, European Comission: Directorate-general Justicie, Freedom and Security (existe traducción en castellano y catalán realizado por la Delegación de Madrid y Barcelona de la Comisión Europea).

Modood, T., Zapata-Barrero, R. y Triandafyllidou, A. (eds.) (2005) Multiculturalim, Muslims and Citizenship: a Europeam approach Londres: Routledge.
Moreras, J. (2003), "Limits and contradictions in the legal recognition of Muslims in Spain", in W. Shadid-P.S. Von Koningsveld (eds.), Religious Freedom and the Neutrality of the State: The Position of Islam in the European Union. Leiden: Peeters.

Nagel, Th. (1991) Equality and partiality, Oxford University Press.

Pérez-Díaz, V., Álvarez-Miranda, B. y Chuliá, E. (2004) La inmigración musulmana a Europa: turcs a Alemanya, algerians a França i marroquins a Espanya, Barcelona: Fundació "La Caixa» (Colecció Estudis Socials, num. 15).

Perrinau, P. (2001) Les croisés de la société fermée, Paris: Editons l'Aube.

Roque, M. A. (2003) El islam plural, Barcelona: Icaria. Solanes, Á. (2004) "Las políticas holísticas de integración de los inmigrantes: su dimensión jurídican, Actas del IV Congreso sobre la Inmigración en España (Noviembre, Girona).

Soysal, Y. N. (1994) Limits of citizenship: migrants and postnational membership in Europe, Chicago and London: The University of Chicago Press.

Süssmuth, R. y Weidenfeld, W. (eds.) Managing Integration: the European Union's responsabilities towards immigrants, Verlag Bertelsmann Stiftung, Gütersloh, 2004. (CD-Rom).

Taggard, P. (2000) Populism, Buckingham: Open University Press.

Taguieff, P. A. (ed.) (2004) Le retour du populisme, Paris: Universalis.

Zapata-Barrero y A. Triandafyllidou, Multiculturalim, Muslims and Citizenship: a Europeam approach Londres: Routledgecon (En prensa).

Zapata-Barrero, R. (2002) El turno de los inmigrantes: esferas de justicia y políticas de acomodación. Imserso, Ministerio de Trabajo y Seguridad Social.

Zapata-Barrero, R. (2003) "State-based logic versus EU-based logic towards immigrants: evidences and dilemman,, Asian and Pacific Migration Journal Vol11, n. 4; 505-528 (traducción de "Fundamentalismo estatal de la UE en torno a la inmigración", Afers Internacionals/Cidob, 53; 149-176).

Zapata-Barrero, R. (2004) Multiculturalidad e inmigración, Madrid: Ed. Síntesis.
Zapata-Barrero, R. (2005) "The Muslim community and Spanish tradition: Maurophobia as a fact, and impartiality as a desideratum", en T. Modood, R.

\section{Documentos oficiales}

Programa de la Hague en Conclusiones de la Presidencia-Bruselas, 4 y 5 de noviembre de 2004.

Comisión Europea, Libertad, seguridad y justicia para todos, Europa en Movimiento, 2004

Directiva 2003/109/CE del Consejo, de 25 de noviembre 2003, relativa al estatuto de los nacionales de terceros países residentes de larga duración.

Directiva 2003/86 CE del Consejo, de 22 de septiembre 2003, sobre el derecho a la reagrupación familiar.

COM (2000) 757final, de 22 de noviembre Comunicación de la Comisión al Consejo y al Parlamento Europeo sobre una política comunitaria de inmigración.

COM (2003) 336 final, de 3 de junio, Comunicación de la Comisión al Consejo, al Parlamento Europeo, al Comité Económico y Social Europeo y al Comité de la Regiones, sobre inmigración, integración y empleo.

COM(2004) 401 final, de 2 junio, Comunicación de la Comisión al Consejo y al Parlamento europeo: Espacio de Libertad, Seguridad y Justicia: balance del programa de Tampere y futuras orientaciones.

ARBOR CLXXXI 713 MAYO-JUNIO [2005] 101-114 ISSN: 0210-1963 